\title{
Deformation measurements of blast loaded plates using digital image correlation and high-speed photography
}

\author{
K. Spranghers ${ }^{1, a}$, D. Kakogiannis ${ }^{1}$, J-M. Ndambi ${ }^{2}$, D. Lecompte ${ }^{2}$, and H. Sol ${ }^{1}$ \\ 1 Faculty of Applied Sciences, Department of Mechanics of Materials and Constructions, Vrije Uni- \\ versiteit Brussel (VUB), Pleinlaan 2, B-1050 Brussels, Belgium \\ 2 Civil and Materials Engineering Department, Royal Military Academy (RMA), Av. De la Renais- \\ sance 30, B-1000 Brussels, Belgium
}

\begin{abstract}
This paper presents a study on the behaviour of aluminium plates subject to close range blast loads. Measurement of the full out-of-plane displacement field is performed combining two high-speed cameras in a stereoscopic set up and the digital image correlation technique. The measured displacement fields are compared to the calculated data using two different FEM codes. A good agreement has been found between both experimental and numerical data.
\end{abstract}

Key words. 3D digital image correlation, high-speed cameras, blast loading, laboratoryscale experiments, thin plate deformation

\section{Introduction}

An explosion in air is a process by which a rapid release of energy generates a pressure wave of finite amplitude. The result is a shock front moving supersonically, with discontinuities in pressure, density, and particle velocity across the front. The shock wave causes an almost instantaneous increase in pressure on nearby objects to a maximum value. Accurate measurement and realistic simulation of problems involving structures under blast loads presents a variety of challenges and is important for the design process.

The response of structural components subjected to blast loading has been the subject of considerable research effort [1-4]. Much of this research has concentrated on the response of plates to impulsive loads [5-9]. These studies deal with large plastic deformations retrieving experimentally the impulse and comparing with analytical and numerical calculations, also the full field response of the specimens was investigated only numerically.

In the present study the full field of displacements, developed during the blast load on aluminium plates, is measured with the digital image correlation technique and compared with two types of finite element simulations.

\section{3D high-speed digital image correlation}

Full-field measurement techniques and the digital image correlation technique (DICT) in particular are very suitable for loading conditions which create complex heterogeneous deformation fields. 2D digital image correlation (2D-DIC) is an optical numerical full-field measuring technique, which offers the possibility to determine in-plane displacement fields at the surface of objects under any kind of loading, based on a comparison between images taken with a digital camera at different load steps.

a e-mail: ken.spranghers@vub.ac.be

This is an Open Access article distributed under the terms of the Creative Commons Attribution-Noncommercial License 3.0, which permits unrestricted use, distribution, and reproduction in any noncommercial medium, provided the original work is properly cited. 
The DICT has been developed in the 80's and has since then extensively been evaluated and improved, however, the fundamental principles of the method remain unchanged and are well described in [10]. By combining stereo-vision principles with 2D-DIC concepts and two synchronised high-speed digital cameras it is possible to measure three-dimensional (3D) deformations fields at high strain rate [11]. The DICT allows studying qualitatively as well as quantitatively the mechanical behaviour of materials under certain loading conditions. Each picture taken with a digital camera corresponds to a different load step. Two images of the specimen at different states of deformation are compared by using a pixel and its signature in the undeformed image, and searching for the pixel in the deformed image in order to maximize a given similarity function. In most cases, this function is based on a least-squares formulation. The signature of a pixel can be anything that discriminates it among any other pixel signature and can be the pixel grey-value, the grey-value derivatives or the pixel colour. In this case, the pixel grey-value is used. A single grey-value is not a unique signature of a pixel; hence, neighbouring pixels are used in practice. Such a collection of pixels is called a subset. The displacement result, expressed in the centre point of the subset, is an average of the displacements of the pixels inside the subset. The step size defines the number of pixels over which the subset is shifted in $\mathrm{x}$ - and y-direction to calculate the next result. Both parameters are expressed in pixels. The uniqueness of each signature is only guaranteed if the surface has a non-repetitive, isotropic, high-contrast pattern. Random textures of speckle patterns fulfill this constraint and can be obtained by an arbitrary speckle pattern that is applied onto the object surface, or that is offered by the texture of the specimen's material. Possible matches at several locations are checked and a similarity score (correlation function) is used to grade them. A classic correlation function using the sum of the squared differences of the pixel values is used. The image correlation routine allows locating every subset of the initial image in the deformed image. Subsequently, the software determines the displacement values of the centres of the subsets, which yields an entire displacement field (relative to the considered reference image).

3D digital image correlation (3D-DIC) uses the same correlation concepts as described above. Two synchronised cameras coupled with stereo-vision principles are required to obtain three-dimensional displacement fields. The manipulation of the stereo-vision system is described in detail in section 3 .

\section{Experimental setup and procedure}

The experimental setup is shown in Fig. 1 and located at the test bunker of the Laboratory of Analysis of Explosion Effects (LAEE) of the Royal Military Academy (RMA). The setup consists of a steel frame (mounting plate, $1 \mathrm{~m} \times 1 \mathrm{~m} \times 1.2 \mathrm{~cm}$ ) with a square captivity of $30 \mathrm{~cm}$ by $30 \mathrm{~cm}$ in the centre. The specimen, a thin aluminium plate (type EN AW-1050A), of $40 \mathrm{~cm}$ by $40 \mathrm{~cm}$ with a thickness of $3 \mathrm{~mm}$, is connected to the steel frame. The connection is established by using a bolts connection with a steel plate in order to achieve a perfect fixed connection. The explosive material, $40 \mathrm{~g}$ of $\mathrm{C} 4$, is hung up behind the test plate with a stand-off distance of $25 \mathrm{~cm}$ from the centre point by using the ignition wires and the detonator in order to realize a spherical airburst wave. A blast pencil and a laser distance meter (range: $400 \mathrm{~mm}$ ) respectively measure the incoming pressure wave and the centre displacement of the test plate. Both measure at a frequency of $1 \mathrm{MHz}$. Two similar Photron@C Fastcam Ultima APX-i2 high-speed digital cameras are mounted in a stereo configuration to record synchronized images during a free-air blast event. The full-field transient deformations of a thin aluminium plate are obtained using 3D digital image correlation (3D-DIC).

The procedure used to perform the experiment is as follows. First, the aluminium test plate is painted with white paint, a black speckle pattern is printed on the specimen to obtain high contrast images and then bolted together with the steel clamping plate onto the steel mounting plate in his final position. Second, the laser distance meter is clamped on a steel bar at $60 \mathrm{~cm}$ in front of the test plate at the same height of the plate's centre point, the charge is hang up at $25 \mathrm{~cm}$ stand-off distance behind the test plate and the blast pencil is positioned in a way the pressure sensor measures the same incident pressure as the plate's centre point. Third, the high-speed cameras are placed in front of the plate (see Fig. 1(a)) at a certain object distance $L_{0}$ from the detonation point to avoid camera motions induced by the blast loading, rotated to view the specimen and maximize the common field of view of the specimen ( $\alpha$ is the angle between the two cameras) and focused. Since large out-of-plane displacements are expected 


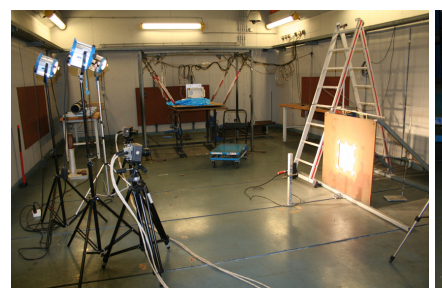

(a)

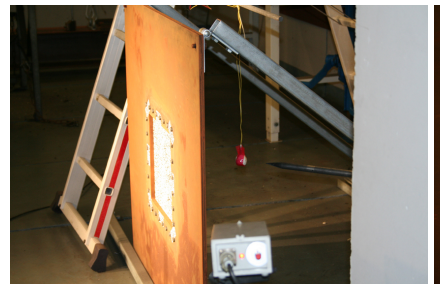

(c)

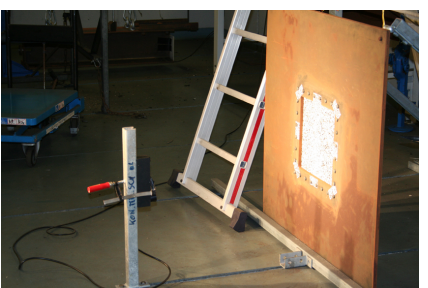

(b)

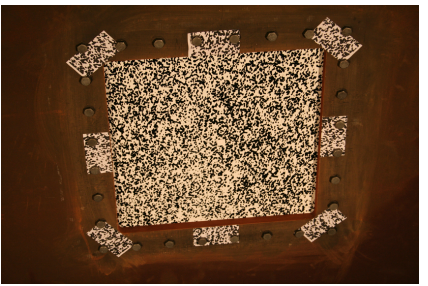

(d)

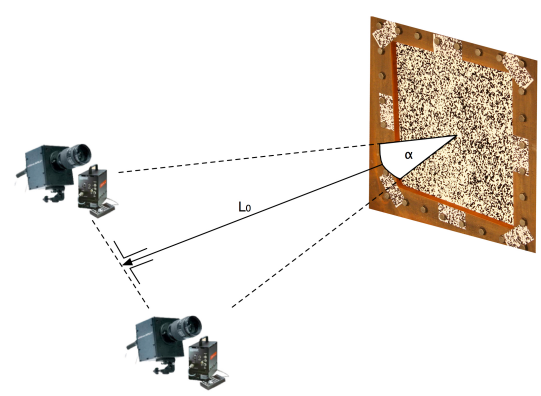

(e)

Fig. 1. Photos of the experimental setup: (a) global view of the setup with the steel mounting plate, two highspeed digital cameras mounted in a stereo configuration, the laser distance meter and three light spots; (b) laser distance meter and steel mounting plate; (c) explosive charge, blast pencil and light intensity trigger; (d) clamped aluminium test plate painted with a black and white speckle pattern; and (e) object distance $L_{0}$ and angle $\alpha$ between the two cameras.

during the deformation process, the aperture of both lenses is reduced and the sheet illumination is increased to compensate for the reduced aperture and maintain adequate contrast throughout the experiment. These settings ensure that the formed sheet remains in focus. Fourth, a calibration panel is used to calibrate the high-speed stereo-vision system. Fifth, a light intensity trigger is placed near the detonation point and is adjusted in a way it will be triggered by the bright light flash of the explosion and send this starting signal to the measurement devices (high-speed cameras, laser distance meter and blast pencil). As soon as the explosion initiates, synchronized stereo images at a frame rate of $6000 \mathrm{fps}$ are acquired during sheet deformation. During the experiments the total plate behaviour is observed with a field of view of $500 \mathrm{~mm} \times 500 \mathrm{~mm}$ and an image size of $512 \times 512$ pixels.

To ensure the accuracy of the results, the stereo-vision system is calibrated before each of the experiments as described by Tiwari et al. in [11]. The calibration panel consists of a grid of circular dots arranged in a pre-determined pattern. During the calibration this panel is moved (translated and rotated) in and out of plane and several images are acquired by both of the cameras. Once the images are acquired, coordinates of the centre of the dots in the calibration images are extracted using suitable edge detection method for all the calibration images for both the cameras. As the placement and inter-spacing of the dots in a calibration grid are pre-determined, using the knowledge of coordinate positions extracted from the calibration grid images a correspondence is drawn up between the coordi- 
nate system of both the cameras. This information is later used for feature based image registration (a process of transforming images of the object from different perspectives or at different time into one coordinate system) and measuring surface profile deformations. Once the camera calibration process is completed, actual digital image correlation is performed using undeformed and deformed image pairs to match common image subsets within the speckle patterns. Once an image subset is matched between undeformed and deformed image pairs, the stereo-vision system parameters are used to estimate the 3D position of points on the undeformed sheet (initial profile) and also on each deformed sheet position. Both stereo calibration and 3D image correlation are performed using Vic3D software developed by Correlated Solutions, Incorporated [12].

\section{Experiments and results}

Two types of experiments were performed. For the first set of tests the aluminium is plate simply hang up by two bolds at the top without fixing them in order to move the plate by hand. This rigid body displacement is measured both with the cameras as the laser distance meter. By comparison of the plate's centre point displacement the accuracy is evaluated. In the second set of tests the aluminium test plate is subjected to blast loading as described in section 3. Full-field 3D surface displacements were determined during blast loading of the aluminium sheet, with the main objective of evaluating displacement measurements of thin plates subjected to blast loading using high-speed digital cameras and 3D digital image correlation.

\subsection{Rigid body displacements}

Two sets of tests are performed. In both cases we use the two high-speed digital cameras and the laser distance meter, respectively at 50fps and $1 \mathrm{kHz}$. Based on the images $(512 \times 512$ pixels) captured by the cameras the displacement of every point is calculated with the 3D-DIC. From this full-field displacement field, the out-of-plane centre point displacement of the plate is extracted and compared with the displacement measured by the laser distance meter.

The displacement of the plate's centre point measured by the two systems agrees very well. The average displacement error of the 3D-DIC and the LDM equals respectively to $0.03 \mathrm{~mm}$ and $0.33 \mathrm{~mm}$.

\subsection{Blast loading}

Two blast load tests are performed as explained in section 3. The object distance $\left(L_{0}\right)$ and angle $(\alpha)$ between the two cameras are respectively $2.5 \mathrm{~m}$ and $39.6^{\circ}$ (see Fig. 1(e)). In the blast load test the cameras capture synchronised images at $6000 \mathrm{fps}$ and the laser distance meter measures the centre point out-of-plane displacement at $1 \mathrm{MHz}$. From the full-field displacement field, the out-of-plane centre point displacement of the plate is extracted.

To obtain three dimensional full-field displacement fields (a) an area of interest (aoi) of $250 \times 250$ pixels, (b) a $33 \times 33$ pixel subset and (c) a subset spacing of 3 pixels is selected. Subset matching is performed using an affine subset shape function.

Error assessment is based on the output variable $\sigma$, which is calculated by the correlation software and represents the confidence interval for the match of a certain point given in pixels. The displacement error, $\varepsilon_{\sigma}$, is equal to the maximum $\sigma$ value of all points for every image multiplied by the image resolution given in millimetres per pixels.

As noticed in previous experiments, the steel mounting frame (see Fig. 1) vibrates due to the explosion impact, which results in additional displacement components in the full-field displacement field of the aluminium test plate. The mounting frame is provided with a speckle pattern at certain points as shown in Fig. 1(d) to take into account these additional displacements of the mounting frame. By subtracting the out-of-plane displacement of the centre of the mounting frame from the out-of-plane displacement given by the 3D-DIC we obtain the pure out-of-plane displacement of the aluminium test plate. 
The out-of-plane displacement of the aluminium test plate's centre point during the blast impact for test 1 and 2 is shown in Fig. 4. The full out-of-plane displacement field at maximum deformation is shown in Fig. 2 and Fig. 3, respectively.

The error for the out-of-plane displacement equals to $\varepsilon_{\sigma}=0.022 \mathrm{~mm}$ and $\varepsilon_{\sigma}=0.019 \mathrm{~mm}$ for blast experiment 1 and 2, respectively. It is worthless to compare the results from the 3D-DIC with the outof-plane displacement measured with the laser distance meter because the laser measurements from the blast experiments are not accurate.

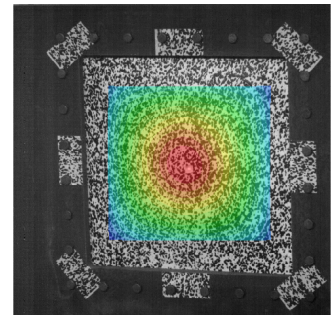

(a) $2 \mathrm{D}$
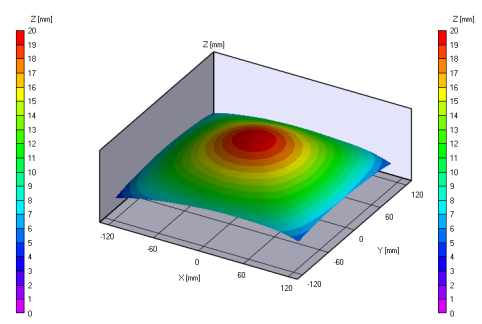

(b) $3 \mathrm{D}$

Fig. 2. Blast impact test 1: full-field displacement of the aluminium test plate. Maximum out-of-plane deformation at $t=0.83 \mathrm{~ms}$.

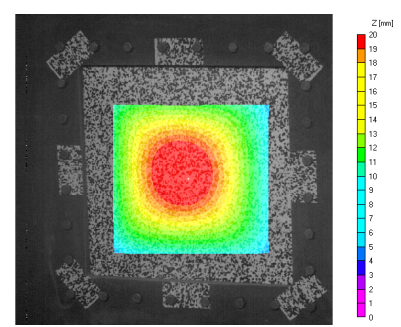

(a) $2 \mathrm{D}$

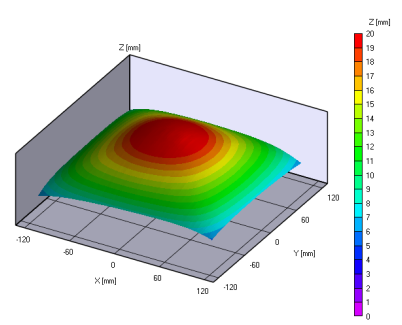

(b) $3 \mathrm{D}$

Fig. 3. Blast impact test 2: full-field displacement of the aluminium test plate. Maximum out-of-plane deformation at $t=1.00 \mathrm{~ms}$.

\section{Numerical study}

The numerical methods used to simulate the blast effects problem are typically based upon a finite volume, finite difference or finite element method with an explicit time integration scheme. In this paper both hydrocodes LS-Dyna and AutoDyn are used to predict the blast loading response of a clamped aluminium test plate.

Simulations in LS-Dyna are based on an empirical method that is developed by Kingery and Bulmash [16] where airblast parameters from spherical air bursts and from hemispherical surface bursts are predicted by equations. These equations are widely accepted as engineering predictions for determining free-field pressures and loads on structures. The Kingery-Bulmash equations have been automated in the computer program ConWep [17]. Curve-fitting techniques are used to represent the data with high-order polynomial equations, assuming an exponential decay of the pressure with time:

$$
P(t)=P_{\text {inc }}\left[1-\frac{t-t_{a}}{t_{+}}\right] \exp \left[-\frac{a\left(t-t_{a}\right)}{t_{+}}\right]
$$


The objective of this algorithm is to produce an appropriate pressure history given an equivalent TNT explosive weight. The quantities to be determined by the algorithm are: $P_{i n c}$ the peak incident pressure; $P_{\text {ref }}$ the peak reflected pressure; $t_{a}$ the time of arrival of the shock wave; $t_{+}$the positive phase duration; $a, b$ the exponential decay factors for incident and reflected waves, respectively. The input values that need to be chosen are: the amount of explosive charge; $R$ the range from charge location to the centroid of loaded surface; and $\cos \theta$ the $\operatorname{cosine}$ of the incident angle, angle between surface normal and range unit vector.

In the idealized case of ConWep there is no decay coefficient and the pressure wave is considered as a special triangular impulse because the structure is considered to be rigid and its surface infinite. ConWep is implemented in LS-Dyna by Randers-Pehrson and Bannister and takes into account the decay coefficient which also updates the pressure history based on changes in the geometry $[13,14]$. The parameters that need to be defined by the user are the TNT - equivalent mass of the explosive and the position of the center of the explosion in space, in order the stand off distance to be defined.

In the LS-Dyna simulation, the Cowper-Symonds [18] constitutive relationship is employed to estimate the strain rate effects on yield stress of the aluminium alloy [11].

$$
\frac{\sigma_{0}^{\prime}}{\sigma_{0}}=1+\left(\frac{\dot{\varepsilon}}{D}\right)^{1 / q}
$$

$\sigma_{0}^{\prime}$ is the dynamic yield stress at a uniaxial plastic strain rate $\dot{\varepsilon}, \sigma_{0}$ is the associated static yield stress and $D$ and $q$ are constants for a particular material. The EN AW-1050A aluminium plate specimen has a static yield stress of $\sigma_{0}=83 \mathrm{MPa}$. The Cowper-Symonds model parameters for EN AW-1050A are assumed to be those given by Bodner and Symonds for alluminium alloys [4,19], $D=6500 \mathrm{~s}^{-1}$ and $q=4$. It should be noted that these model parameters were determined using uniaxial dynamic loading conditions, with insufficient data available to improve parameter estimates for material behaviour under dynamic biaxial loading conditions [11].

In LS-Dyna the Cowper-Symonds model is integrated in Material Type 3: Kinematic/Isotropic Elastic/Plastic [14]. For the EN AW-1050A aluminium plate specimen following model parameters are used: material density $\rho=2706 \mathrm{~kg} / \mathrm{m}^{3}$; Young's modulus $E=69 \mathrm{GPa}$; strain rate parameter $C=$ $6.5(m s)^{-1}$ (cfr. $\left.D\right)$; strain rate parameter $p=4(m s)^{-1}$ (cfr. $q$ ); Poisson's ratio $v=0.33$; and yield stress $\sigma_{0}=83 \mathrm{MPa}$.

The numerical simulation is also performed using Autodyn-3D [15]. The simulation is carried out in two steps: (a) the simulation of the blast wave propagation in the air, and (b) the analysis of the interaction of the air blast with the aluminium plate. These two steps are necessary in order to minimize the numerical run-time of the simulation. For air, density, pressure cut-off and viscosity coefficient are defined. The viscosity and pressure cut-off are set to zero, because pressure cannot be negative and the viscosity forces are negligible. The ideal gas law is used as the equation of state for air. For the explosive and its detonating products, the Jones-Wilkins-Lee (JWL) equation of state (EOS) relating energy, pressure and density is applied. While there are various types of EOS describe the state of detonation products, the JWL-EOS is widely used because of its simplicity. For the aluminium plate we used the linear material EOS and the Steinberg-Guinan [20] constitutive relationship, which are both integrated in the material library of AutoDyn [15].

The comparison of the out-of-plane plate's centre point between the experiments and finite elements simulations is shown in Fig. 4. Here we can see that the calculated out-of-plane displacement curves follow the experimental displacement curves. Both experimental and numerical curves have a maximum plastic deformation in the first peak, followed by elastic deformations and damping. In the numerical calculations no damping is used.

The maximum displacements and the displacement after blast loading (plastic deformation) are listed in Table. 1. The AutoDyn simulation is most consistent with the experimental data. Both LS-Dyna and Autodyn can be used to predict the maximum centre point's out-of-plane displacement. The simulation time in AutoDyn is thousand times slower than the LS-Dyna using ConWep, however the AutoDyn simulation gives better results. 


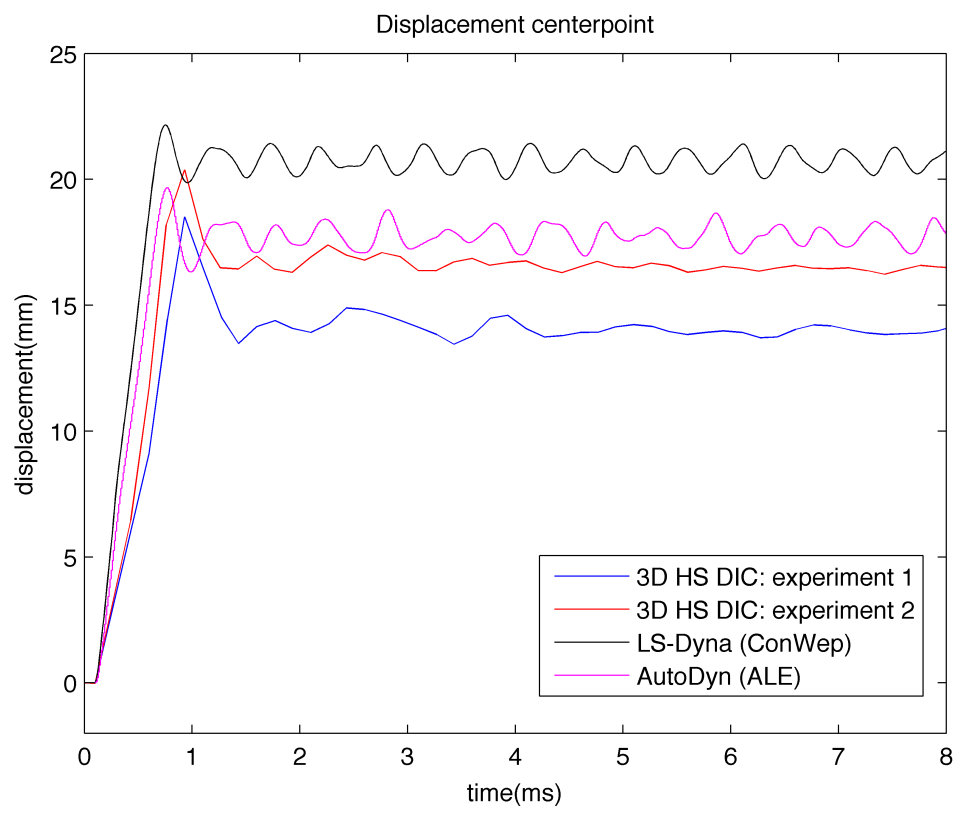

Fig. 4. Comparison of experimental blast impact test 1 and 2 with numerical blast simulation in LS-Dyna and AutoDyn; displacement plate's centre point.

Table 1. Comparison of experimental blast impact test 1 and 2 with numerical blast simulation in LS-Dyna and AutoDyn; maximum displacement and plastic deformation.

\begin{tabular}{c|lllc}
\hline & $\begin{array}{l}\text { 3D HS DIC } \\
\text { experiment 1 }\end{array}$ & $\begin{array}{l}\text { 3D HS DIC } \\
\text { experiment 2 }\end{array}$ & LS-Dyna & AutoDyn \\
\hline maximum displacement (mm) & 18.51 & 20.38 & 22.16 & 19.66 \\
plastic deformation (mm) & 14.07 & 16.58 & 20.72 & 17.74 \\
\hline
\end{tabular}

\section{Conclusions}

High-speed stereo vision with 3D Digital Image correlation has been successfully used to measure the rigid body displacement of a plate specimen. The calculated out-of-plane displacement is evaluated by the comparison with a laser distance meter and the estimated accuracy equals to $0.03 \mathrm{~mm}$ which is sufficient and in the order of magnitude of the plate specimens' geometrical imperfections.

More important this high-speed stereo vision system has been successfully used to monitor dynamic specimen response during free-air blast loading with a frame rate of 6000fps and an estimated accuracy on the out-of-plane displacement of $0.022 \mathrm{~mm}$. Results show that free-air bast loading induces highly localised, rapid material response. Using well-known relationships, the material response is simulated with two different methods which both give comparable results with the experimental data from the 3D-DIC. On the other hand, out-of-plane displacements measurements during blast impact using a laser distance meter as used in this setup do not give correct data, same applies to the measurement of the incident pressure in the centre of the plate using a blast pencil.

As a conclusion, the results confirm that dynamic stereo-vision systems are capable of accurately measuring surface displacement and deformation data at high rates, providing investigators with the ability to study a wide range of dynamic events, including impact, penetration and blast loading. 


\section{References}

1. P.S.Bulson, Explosive loading of engineering structures: a history of research and review of recent developments (E\&FN SPON, UK 1997) p. 236

2. E. Yandzio, M. Gough, Protection of buildings against explosions (Steel Construction Institute, UK 1999) p. 110

3. M. Y. H. Bangash, T. Bangash, Explosion-resistant buildings: design, analysis, and case studies (Springer, USA 2006) p. 784

4. N. Jones, Structural Impact (Cambridge University Press, UK 1989) p. 575

5. R. G. Teeling-Smith, G. N. Nurick, International Journal of Impact Engineering 11, (1991) p. 77-91

6. G. N. Nurick, G. C. Shave, International Journal of Impact Engineering 18-1, (1996) p. 99-116

7. M. D. Olson, G. N. Nurick, J.R. Fagnan, International Journal of Impact Engineering 13-2, (1993) p. $279-291$

8. A. Neuberger, S. Peles, D. Rittel, International Journal of Impact Engineering 34, (2007) p. 859-873

9. A. Neuberger, S. Peles, D. Rittel, International Journal of Impact Engineering 34, (2007) p. 874-882

10. M. A. Sutton, J. -J. Orteu, H. W. Schreier, Image Correlation of Shape, Motion and Deformation Measurements: Basic Concepts, Theory and Applications (Springer, USA 2009) p. 321

11. V. Tiwari, M. A. Sutton, S. R. McNeill, S. Xu, X. Deng, W. L. Fourney, D. Bretall, International Journal of Impact Engineering 36, (2009) p. 862-874

12. Correlated Solutions Incorporated, VIC-3D (USA, www.correlatedsolutions.com)

13. G. Randers-Pehrson, K. A. Bannister, Airblast Loading Model for DYNA2D and DYNA3D (Report ARBL-TR-1310, U.S. Army Ballistic Research Laboratory, USA 1997)

14. Livermore Software technology Corporation, LS-DYNA User's Manual: Nonlinear Dynamic Analysis of Structures (Version 950) (USA 1999) p. 1024

15. Century Dynamics Incorporated, AUTODYN-3D User's Manual (USA 1990)

16. C. N. Kingery, G. Bulmash, Airblast Parameters from TNT Spherical Air Burst and Hemispherical Surface Burst (Report ARBL-TR-02555, U.S. Army Ballistic Research Laboratory, USA 1984)

17. U.S. Department of the Army, Fundamentals of Protective Design for Conventional Weapons (Department of the Army Technical Manual, USA 1986)

18. G. Cowper, P. S. Symonds, Strain-hardening and strain-rate effects in the impact loading of cantilever beams (Division of Applied Mathematics, Brown University, USA 1957) p. 46

19. S. R. Bodner, P. S. Symonds, Journal of Applied Mechanics 29, (1962) p. 719-728

20. D. J. Steinberg, S. G. Cochran, M. W. Guinan, Journal of Applied Physics 51, (1980) p. 1498-1504 